\title{
A Review of the Application of Fractal in Recognition
}

\author{
YongqinYuan 1, a, Fengming Liu, b, Chuiyun Zhou ${ }^{2, c}$ \\ ${ }^{1}$ School of Management Science and Engineering, Shandong Normal University, Ji'nan 250014, \\ China \\ 2Shandong Polytechnic, Ji'nan 250014, China \\ ayongqinyuan1993@163.com, bfmliucn@gmail.com,c393551606@QQ.com
}

Keywords: fractal; identification; information.

\begin{abstract}
Based on the fractal theory, this paper briefly reviews the origin and definition of fractal theory, and the concept of fractal dimension and its main calculation method. Then select one of the applications of fractal theory, in the application of recognition, to have a literature review. This paper summarizes the domestic and foreign research literature in recent 5 years, it is found that fractal recognition is involved in image, signal and waveform, but there is no breakthrough in the recognition of text information. Therefore, this paper proposes to use fractal ideas for text information recognition.
\end{abstract}

\section{Introduction}

Fractal theory was originally used to study the coastline, then it is developed into a theory that study the development and change of things. At present, fractal theory is not only used to study the coast, water, landform, but to complex networks, domestic urban planning, capital markets, agriculture and food systems, oil and gas storage and transportation, stock market, image coding and other fields. In addition, the application of fractal theory in recognition is also gradually highlighted, which mainly includes image recognition, signal recognition and so on. Therefore, from another point of view, this article reviewed the study of fractal theory in the identification, and put forward a new idea that the fractal principle can be applied to the information recognition aspects.

\section{Fractal Theory Introduction}

\subsection{Sources and Definitions of Fractal.}

How long of "British coastline"[1], published by the famous American mathematical scientist B.B.Mandelbrot, in 1967, which marks the emergence of fractal theory. In 1975, in the "fractal: shape, opportunity and dimension" Mandelbrot formally put forward fractal geometry, contrast to the traditional European geometric form, thus creating a new stage of fractal geometry.

Fractal - Partially shaped in some form like the whole is called a fractal (B.B.Mandelbrot).

A fractal set is a set that is more irregular than any collection of traditional geometric studies, whether it is magnified or reduced, and the irregularity of the set is still evident. The basic characteristics of fractal can be summarized as following:

1. Fine structure - with irregular dimensions of any scale

2. Difficult to describe - its irregularity cannot be described locally and in the traditional geometric language

3. Self-similarity (scale invariance) - local and integral with some self-similarity

4. Fractal dimension is greater than the topological dimension

5. It may be generated by iteration

\subsection{Fractal Dimension Theory.}

The dimension is used to describe the position of an object in space, or the minimum number of coordinates required to determine the position of the geometric object in space. For the fractal set we discuss, we also need to use dimension to describe its characteristics, that is, fractal dimension ${ }^{[2]}$. The biggest difference between fractal dimension and continental dimension and topological dimension is that the fractal dimension can be a fraction, and the latter can only be an integer. Fractal dimensions 
reflect the irregular degree of the graph, the larger the value is, the less irregular the graph is. Among the common dimensions are the Similarity Dimension, the Box Dimension and the Hausdorff Dimension ${ }^{[3]}$. Fractal dimensions play a very important role in the application of fractal, and the self-similarity and its related properties can be explored by solving the fractal dimension of some special graphs.

\section{Fractal Theory in Various Fields on the Application of Identification}

Since fractal theory is put forward, fractal as a new method of analyzing problems is widely used in various fields. At the same time, the application of recognition emerges in endlessly, the following summarizes the nearly five years of fractal theory in various fields on the identification of the study.

\subsection{Computer Engineering and Applications.}

In the field of computer engineering and application, fractal recognition is mainly applied to image recognition, face recognition, emotion recognition and speech recognition. In the application of image recognition, fractal theory is usually used in conjunction with image segmentation.

Using fractal theory for image recognition, different scholars have different research methods. Among them, some scholars use the idea of segmentation into the graphics pretreatment, fractal and segmentation are been combined. Haijing $\mathrm{Yu}^{[4]}$ combines the fractal theory with the color image for smoke recognition, combining the fractal segmentation method with the color image segmentation method to identify the smoke. Qingtian Geng et al. ${ }^{[5]}$ based on the fractal dimension of the license plate binarization processing, proposed a fractal dimension and hidden Markov feature based license plate recognition algorithm. Le Dai ${ }^{[6]}$ uses multiple fractal and improved BP algorithms to improve image recognition accuracy. Benouis Mohamed et al. ${ }^{[7]}$ proposed a new method of extracting features and facial recognition by using the fractal code determined by fractal coding. Xiaojuan Chen et al. ${ }^{[8]}$ used fractal for image feature extraction for face recognition, and after splitting the image, the fractal dimension of each image sub-block was taken as the eigenvector of the image. Yunhua Chen ${ }^{[9]}$ analyzes the application of fractal coding in face recognition, and studies the current fractal technique for face recognition. Zhijie Tang et al. ${ }^{[10]}$ proposed a new face recognition method called Fractal Neighbor Distance Classification (FNDC). Hossein Pazhoumand-dar et al. ${ }^{[1]}$ proposed a new method for detecting, tracking and identifying traffic signs by processing candidate blocks of the tracking module to extract their fractal characteristics.

There are some scholars who focus on the texture features of the research department's images, including texture, roughness, and edge features. Chengsheng Yuan ${ }^{[12]}$ proposed to apply the fractal dimension into the field of blade recognition, as a method of leaf texture feature extraction to represent the texture complexity and self-similarity of the leaf image, so that the leaf identification can be better effect. P. Shanmugavadivu and V. Sivakumar ${ }^{[13]}$ proposed an algorithm for calculating the fractal dimension of digital images, comparing the fractal dimensions of these images and proving that fractal dimension is an ideal tool for measuring image roughness. YingChao Zhang et al. ${ }^{[14]}$ use fractal edge detecting method for extracting edge features of characters, fractal edge feature with three other features (loop region, a projection histogram moment) in combination, in conjunction with a classifier, for recognizing handwritten numerals Methods. JiXiang Du et al. ${ }^{[15]}$ discussed the method of describing plant leaf characteristics based on contour fractal dimension and pulsed fractal dimension, and calculated the two-dimensional fractal dimension of leaf edge image and multiple vein images as the basis for identification. Haiyan Ding et al. ${ }^{[16]}$ consider the statistical law of fractal dimension of characterization of cloud and snow texture, and propose a method of cloud and snow automatic recognition based on fractal dimension. Shoumei Wang ${ }^{[17]}$ proposed two fractal methods to identify the crack information in the road surface damaged image, one is the fractal self-similarity of the road surface damage image recognition, the other is based on fractal theory discrete scores Brownian motion model for identification. In order to distinguish between natural images (NI) and computer-generated graphics (CG), Fei Peng et al. ${ }^{[18]}$ Analyzed the differences in residual images, extracted 9-dimensional and 9-dimensional features of histogram features Its texture difference. Mohammad Reza Faraji and Xiaojun $\mathrm{Qi}^{[19]}$ proposed a new method based on logarithmic function and fractal analysis (FA) to generate logarithmic fractal dimension (LFD) images of illumination invariants for face recognition. 
Synthetically, fractal recognition in computer engineering applications are mainly in the image recognition, including the most widely used face recognition, there are some other image recognition. In the whole process of image recognition, fractal theory is mainly applied to feature extraction. Based on the method of image recognition, it can be divided into segment recognition and texture recognition. Segment recognition usually have image dividing at first, each image is divided into several rational sub-blocks, then calculate the fractal dimension of each sub-blocks. The fractal dimension is taken as part of the eigenvector, and combined with other features to classify the classification algorithm.

Texture recognition mainly combine the image shape, area, gray edge characteristics and other factors, for fractal dimension calculation (genetic algorithm, particle swarm algorithm, blanket fractal, multiple fractal, etc.), and then extract the feature vector to construct classifier.

\subsection{Telecommunications Technology.}

Fractal applications in the field of telecommunications technology can also be divided into two categories: signal identification and pattern recognition.

There are many studies on signal recognition. FangyueDang et al. ${ }^{[20]}$ propose a method to combine the higher order of the signal and the dimension of the fractal box dimensions, extract the fractal box dimension of the signal as a supplement to the higher order cumulant, construct the joint feature parameters and applied to signal modulation identification. Yuanlian $\mathrm{Huo}^{[21]}$ uses the fractal dimension of the ground radiation field signal and the fractal dimension of the lightning to perform the identification of round flash and cloud flash different discharge type. Qizhong Zhang et al. ${ }^{[22]}$ proposed a method of EMG signal pattern recognition based on empirical mode decomposition and multifractal analysis. Xingming Guo ${ }^{[23]}$ proposes a method for identifying heart sounds based on EMD correlation dimension and multifractal spectrum. For the station in steady state. $\mathrm{Yu} \mathrm{Ning}^{[24]}$ uses the correlation dimension to measure the characteristics of the correlation complexity of the signal, and proposes a radar signal recognition algorithm based on fractal theory and neural network. Wentao Liu ${ }^{[25]}$ proposed a new communication signal identification method, by extracting the box dimension and the information dimension of the signal after the pre-processing signal dimension and information dimension, as the identification of the characteristic parameters to achieve unknown signal recognition. Yi-bing Li et al. ${ }^{[26]}$ proposed a recognition algorithm based on multi-scale feature, using multi-fractal dimension to extract the subtle characteristics of different impulse noise signals to achieve the purpose of classification and identify the radiation source. HongChen et al. ${ }^{[27]}$ preprocess the received signal, based on the generalized dimension and multifractal spectrum of the signal obtained from the two-dimensional data array, propose a new method of digital communication signal modulation based on multi-fractal feature. Zhiling Tang et al. ${ }^{[28]}$ proposed a signal-based specific communication emitter identification method, extract fractal features from steady-state signals. Takumi Sase et al. ${ }^{[29]}$ estimate the level of dynamic noise by calculating the fractal dimension of the time series to estimate the dynamic noise level of the "short" time series.

Pattern recognition studies are also involved. According to the analysis of the principles of marginal and multifractal theory, Lilong Qin ${ }^{[30]}$ proposed a new feature extraction method based on multiple fractal theory to improve the accuracy of digital signal modulation pattern recognition at low signal to noise ratio. Yang Lu et al. ${ }^{[31]}$ increase the marginal spectrum and multiple fractal modulation mode in the modulation pattern recognition application.

In summary, in the field of telecommunications technology, the identification of identification is mainly in signal recognition and pattern recognition. The signal mainly includes communication signal, radio signal, lightning signal, radar signal, heart sound signal, EEG signal. At present, the identification of signal characteristic parameters mainly includes three steps: signal preprocessing, feature extraction and classification and recognition. Feature extraction is the most important link. Fractal theory can describe the irregular shape of the signal well, therefore, it is usually applied to feature extraction, it is found that the extraction of fractal box dimension, correlation dimension, information dimension, generalized dimension and so on as characteristic parameters are for classification and recognition have good recognition effect. 


\subsection{Other fields.}

In addition to the above areas, fractal in Materials, Machinery, and automation ${ }^{[32-34]}$, geology and mining ${ }^{[34]}$, agriculture and forestry ${ }^{[36]}$, biomedical ${ }^{[37]}$,stock finance, respectively, have different implications.

\section{Conclusion and Future}

This paper summarizes the research of the fractal theory in the field for identification in the past five years. There are some commonalities in applying fractal to recognition.

In the field of computer engineering and application, the field of image recognition and face recognition is the mainstream of the research direction. And most of the methods used by scholars are similar, by calculating the fractal dimension of image as a recognition of the eigenvector. Second is the telecommunications technology, where the mainstream is signal recognition. The signal can be decomposed to obtain the modal function of the signal and extract the fractal dimension of its corresponding function image. In addition, there are the applications in materials and geological areas, this aspect is very professional, but the application of fractal is similar.

Consolidating all fields that have been studied in the literature, the fractal theory is applied to the recognition of things such as images, signals, waveforms, textures and so on, but it is rarely involved in the recognition of text information. At present, most of the recognition methods of text information are to extract the keywords, assign the keywords to the corresponding weights, and then form the eigenvectors, and use the classification algorithm to identify the text information. Associated with the wide application of fractal in recognition, imagine that if fractal theory can be used for text recognition, there will be a major breakthrough in the field of fractal recognition.

\section{Acknowledgements}

This work was supported in part by the National Natural Science Foundation of China (No. 61170038, 61472231), and the National Social Science Foundation of China (No. 14BTQ049)

\section{References}

[1]. Mandelbrot B. How Long Is the Coast of Britain? Statistical Self-Similarity and Fractional Dimension. Science, 1967,156:636-638

[2]. Peres Y. Sousi P. Brownian motion with variable drift:0-1 laws, hitting probabilities an Hausdorff dimensions. Mathematical Proceedings Philosophical Society, 2012, 153: 215-234.

[3]. Sase T. Ramirez J. Estimating the level of dynamical noise in time series by using fractal dimensions. Physics Letters A, 2016, $380 ; 115 ; 1-1163$.

[4]. YU Hai-jing. Color Recognition of Color Smoke Based on Fractal Theory. Beijing: Institute of Precision Instruments and Physics, Chinese Academy of Sciences, 2012.

[5]. GENG Q.T., ZHAO H.W. License plate recognition based on fractal dimension and hidden Markov feature. Optical Precision Engineering, 2013, (12): 3198-3204.

[6]. [6] DAI L., WANG K.K., ZHU Ye. Environmental Image Recognition Based on Multifractal and Improved BP Algorithm. Electronic Design Engineering, 2016, (17): 167-170.

[7]. Mohamed B., Kamel B.M., Redwan T., Mohamed S. A Novel Technique For Human Face Recognition Using Fractal Code and Bi-dimensional Subspace. In: Amine A., Bellatreche L., Elberrichi Z., Neuhold E., Wrembel R. (eds) Computer Science and Its Applications. CIIA 2015. IFIP Advances in Information and Communication Technology, vol 456. Springer, Cham

[8]. Chen Y.H., Zhang X.Fractal coding in the face recognition application. China High-tech enterprises, 2017, (03): 57-59. 
[9]. Chen X.J., Wang D.H. Face Recognition Method Based on BEMD and Fractal Dimension. Computer Engineering and Applications, 2016: 1-5.

[10]. Tang, ZJ. Wu, X., Leng, X. et al. A Fast Face Recognition Method Based on Fractal Coding. SIViP (2017). doi:10.1007/s11760-017-1078-7.

[11]. Pazhoumand-dar, H. \& Yaghoobi, M. A new approach in road sign recognition based on fastfractal coding. Neural Comput \& Applic (2013) 22: 615. doi:10.1007/s00521-011-0718-Z

[12]. YUAN C.s., YAO Y.f. Image Recognition Method Based on Fractal Dimension. Computer Engineering and Design, 2012, 33 (2): 670-673.

[13]. P.Shanmugavadivu, V.Sivakumar.Fractal. Dimension Based Texture Analysis of Digital Images. Procedia Engineering, 2012, 38:2981-986.

[14]. Zhang Y., Liu T., Ye X. Handwriting Digit Recognition Based on Fractal Edge Feature and BP Neural Net [J]. In: Jin D., Lin S. (eds) Advances in Computer Science and Information Engineering. Advances in Intelligent and Soft Computing, vol 168. Springer, Berlin, Heidelberg, 2012.

[15]. Du JX. Zhai CM. Wang QP. Recognition of Leaf Image Based on Outline and Vein Fractal Dimension Feature. In: Huang DS., Gan Y., Bevilacqua V., Figueroa J.C. (eds) Advanced Intelligent Computing. ICIC 2011. Lecture Notes in Computer Science, vol 6838. Springer, Berlin, Heidelberg, 2011.

[16]. Ding H.y., Ma L.L., Li Z.Y., Tang L.L... Automatic recognition method of full - color image based on fractal dimension. Remote Sensing Technology and Application, 2013,28 (1): 52-57

[17]. Wang X.M..Study on crack recognition based on fractured road surface image. Chang'an University, 2014.

[18]. Peng F.,Zhou DL.,Long M.,Sun XM.,Discrimination of natural images and computer generated graphics based on multi-fractal and regression analysis. Int. J. Electron. Commun. (AEÜ) 71 (2017) 72-81.

[19]. Mohammad Reza Faraji n ,Qi XJ., Face recognition under varying illuminations using logarithmic fractal dimension-based complete eight local directional patterns. Neurocomputing,199 (2016) 16-30.

[20]. DANG F.Y., XU Q.J., ZHANG J., CHEN X.Application of higher order cumulant and fractal theory in signal modulation recognition. Signal Processing, 2013, (06): 761-765.

[21]. Huo Y.L., Zhang G.S., Lv S.H., Yuan P. Fractal characteristics of lightning and its application in automatic identification. Acta Physica Sinica, 2013, (05): 574-582.

[22]. ZHANG Q.Z., XI X.G., LUO Z.Z... Application of multifractal analysis in EMG pattern recognition. Technical Journal of Sensors and Actuators, 2013, (02): 282-288.

[23]. GUO X.M., ZHANG W.Y., YUAN Z.H., HIS Y.Q., LI Ch.P. Non-tone recognition based on EMD correlation dimension and multifractal spectrum. Journal of Instrumental Analysis, 2014, (04): 827-833.

[24]. Ning Yu. Radar Signal Recognition Algorithm Based on Fractal Theory and Neural Network Small and Medium Enterprise Management and Technology (Zhongxian), 2014, (02): 313-314.

[25]. Liu W.T., Chen H., Cai X.X., Liu J.T... Digital Signal Recognition Based on Fractal Dimension. Fire and Command Control, 2014, (10): 88-90 + 94.

[26]. Li YB.,Nie W., Ye F., Li JC.,A Pulse Signal Characteristic Recognition Algorithm Based onMultifractal Dimension. Mathematical Problems in Engineering, Volume 2014, 6 pages 。 
[27]. Chen Hong, Cai X.X., Xu Y., Liu W.T. Study on Communication Modulation Recognition Based on Multifractal Feature. Journal of Electronics \& Information Technology, 2016, (04): 863-869.

[28]. Tang Z., Li S. Steady Signal-Based Fractal Method of Specific Communications Emitter Sources Identification. In: Zeng QA. (eds) Wireless Communications, Networking and Applications. Lecture Notes in Electrical Engineering, vol 348. Springer, New Delhi, 2016.

[29]. Takumi S., Jonatán P.R.,Keiichi K. , Kazuyuki A. ,Yoshito H., Estimating the level of dynamical noise in time series by using fractal dimensions. Physics Letters A 380 (2016) 1151-1163.

[30]. Qin L.L., Wang Z.Y. Application of marginal spectrum and multifractal in modulation pattern recognition. Journal of Intelligent Systems, 2014, (06): 756-762.

[31]. LU Y., YI P., WANG Xi. Application of marginal spectrum and multifractal in modulation pattern recognition. Information and Communication, 2015, (12): 10-11.

[32]. ZHOU Y.R. Study on classification and classification of metallographic defects in cast aluminum alloy based on fractal theory. Hunan University, 2014.

[33]. Su Tao. Based on the multi-fractal theory of refractory acoustic emission signal feature extraction and damage pattern recognition research. Wuhan University of Science and Technology, 2015.

[34]. Liao, J., Zhang, J., Feng, P. et al. Identification of contact stiffness of shrink-fit tool-holder joint based on fractal theory. Int J Adv Manuf Technol (2017) 90: 2173.

[35]. [35]ZHAO G.Y.n, DENG Qi.L., LI X.B., DONG L.J., CHEN G.H., ZHANG Chu-hong. Characteristics of microseismic waveforms based on EMD and morphological fractal dimension. Journal of Central South University (Natural Science Edition), 2017 (01): 162 -167.

[36]. [36]Salmasi M., Büttner U., Glasauer S. Fractal dimension analysis for spike detection in low SNR extracellular signals. Journal of Neural Engineering, 2016, Vol.13 (3)

[37]. [37] FAN Di, WANG L.X., YANG W.R., TAN K.L., QIU R.Z.. Feature fluorescence spectroscopy based on fractal dimension difference model. Journal of Heilongjiang August First Land Reclamation University, 2016 (04): 130-134. 